ARTICLE

Received 16 Oct 2014 | Accepted 26 Mar 2015 | Published 7 May $2015 \quad$ DOl: 10.1038/ncomms8034

\title{
Charge order from orbital-dependent coupling evidenced by $\mathrm{NbSe}_{2}$
}

Felix Flicker ${ }^{1} \&$ Jasper van Wezel ${ }^{2}$

Niobium diselenide has long served as a prototype of two-dimensional charge ordering, believed to arise from an instability of the electronic structure analogous to the one-dimensional Peierls mechanism. Despite this, various anomalous properties have recently been identified experimentally, which cannot be explained by Peierls-like weakcoupling theories. Here, we consider instead a model with strong electron-phonon coupling, taking into account both the full momentum and orbital dependence of the coupling matrix elements. We show that both are necessary for a consistent description of the full range of experimental observations. We argue that $\mathrm{NbSe}_{2}$ is typical in this sense, and that any chargeordered material in more than one dimension will generically be shaped by the momentum and orbital dependence of its electron-phonon coupling as well as its electronic structure. The consequences will be observable in many charge-ordered materials, including cuprate superconductors.

\footnotetext{
${ }^{1}$ H. H. Wills Physics Laboratory, University of Bristol, Tyndall Avenue, Bristol BS8 1TL, UK. ${ }^{2}$ Institute for Theoretical Physics, Institute of Physics, University of Amsterdam, 1090 GL Amsterdam, The Netherlands. Correspondence and requests for materials should be addressed to F.F. (email: flicker@physics.org) or J.v.W. (email: vanwezel@uva.nl).
} 
T he charge density wave (CDW) order in $2 \mathrm{H}-\mathrm{NbSe}_{2}$ has been surrounded by controversy since its first observation nearly 40 years ago ${ }^{1}$. The most basic unresolved question concerns the driving mechanism of the ordering transition. In quasi-one-dimensional (1D) materials, it is well known that charge order arises from a Peierls instability: any interaction between states at the Fermi energy $\left(E_{\mathrm{F}}\right)$ results in a periodic modulation of both the electronic charge density and the atomic lattice positions, which lowers the free energy through the corresponding opening of a band gap ${ }^{2}$. Such a mechanism is termed weak coupling since an arbitrarily small interaction will drive the system to order at low enough temperatures. In higherdimensional systems, a nesting of the Fermi surface is typically required for such an instability to be effective. In $\mathrm{NbSe}_{2}$, however, Fermi surface nesting does not occur ${ }^{1,3-5}$. The lack of a lock-in transition and the coexistence of CDW order with superconductivity below $7.2 \mathrm{~K}$ nonetheless seem to suggest a weak-coupling origin of the charge order. As a result, a number of alternative weak-coupling mechanisms have been proposed, based on nested saddle-points in the electronic dispersion ${ }^{6}$, local field effects ${ }^{5}$ or a combination of weak nesting with momentum-dependent electron-phonon coupling ${ }^{3,7}$.

There are a number of experimental observations, however, that are hard to reconcile with weak-coupling approaches in $\mathrm{NbSe}_{2}$. The first concerns the size of the electronic gap in the charge-ordered state. Kinks in the density of states (DOS) observed by planar tunnelling experiments have been interpreted to arise from a gap of $\Delta=35 \mathrm{meV}^{8,9}$, while only much smaller gaps between 2 and $5 \mathrm{meV}$ were seen by high-precision angleresolved photoemission spectroscopy (ARPES) experiments ${ }^{4,10}$, and older studies even reported no gap at all in ARPES and resistivity measurements ${ }^{11,12}$. Assuming a weakly coupled driving mechanism for the charge order, the size of the gap is expected to be proportional to the transition temperature, $\Delta \simeq 1.76 k_{\mathrm{B}} T_{\mathrm{C}}$, in direct analogy to the BCS theory of superconductivity ${ }^{13}$. A gap size of $35 \mathrm{meV}$ would then be far too large to explain the observed $T_{\mathrm{CDW}}$ of $33 \mathrm{~K}^{14}$. The $5 \mathrm{meV}$ gap consistent with ARPES data is of the expected size, but it occurs only in isolated points on the Fermi surface ${ }^{4,10}$, raising the question of how the correspondingly small gain in electronic energy can overcome the cost of introducing the periodic lattice distortions associated with CDW order. In addition, while the gaps seen in ARPES measurements are connected by the $\mathrm{CDW}$ wave vector, other equally well-connected points support either only a much smaller gap or no gap at all ${ }^{4,10}$. It was recently suggested, based on scanning tunnelling spectroscopy (STS) measurements ${ }^{15}$, that a particle-hole asymmetric gap of $12 \mathrm{meV}$, centred above $E_{\mathrm{F}}$, exists in $\mathrm{NbSe}_{2}$. The offset in energy explains why ARPES only sees a smaller gap size, while the value of $12 \mathrm{meV}$ is consistent with the order of magnitude expected for a strongly coupled CDW, which may have $\Delta \sim 4 k_{\mathrm{B}} T_{\mathrm{C}}$ in analogy to strong-coupling superconductors ${ }^{16-18}$.

The idea that the CDW order in $\mathrm{NbSe}_{2}$ is of the strong coupling variety, in which a sizable electron-phonon coupling is essential in causing an instability of the electronic structure, is also consistent with two further recent experimental observations. First, it was observed in X-ray scattering experiments that at the transition temperature a broad momentum-range of phonons simultaneously softens towards zero energy $y^{7,19}$. This is in stark contrast to the sharp Kohn anomaly expected in a Peierls-like transition ${ }^{13}$ and may be understood to be an effect of the importance of the entropy carried by phonon fluctuations in the strongly coupled scenario $^{20-22}$. These phonon fluctuations are localized in real space and therefore necessarily involve a broad range of momenta. In an electronically nested system, on the other hand, the nesting vector localizes any fluctuations to a single point in momentum space. The second experimental result arguing for a strongly coupled transition is the observation of a reduction of the density of states persisting well above the transition temperature, in what has been termed a pseudogap regime ${ }^{4,23,24}$. In strongly coupled CDW materials, a pseudogap typically appears through the presence of locally fluctuating charge order without long-range coherence $^{23}$. These same charge fluctuations have recently been suggested to play a central role in the description of the pseudogap phase of cuprate superconductors ${ }^{25-30}$. The precise nature of the pseudogap in these materials, however, is difficult to establish owing to the presence of various nearby competing orders. $\mathrm{NbSe}_{2}$, being free of such complications, provides a test bed on which the formation and characteristics of a pseudogap phase due to charge fluctuations can be studied directly.

Here we present a theoretical analysis of the charge order in $\mathrm{NbSe}_{2}$ based on a model of strong electron-phonon coupling. Although the transition is phonon driven, we find that a full knowledge of the electron states scattered from and to, including both wave vector and orbital dependence, is crucial in explaining the experimental data. Despite a lack of nesting, the limited matching of states at $E_{\mathrm{F}}$ acts in unison with the momentum dependence of the electron-phonon coupling to select out the $\mathrm{CDW}$-ordering vector, while the orbital characters of the bands naturally explain why a CDW gap appears primarily in only one of the Fermi surface pockets. The resulting momentum dependence of the gap itself agrees with ARPES experiments as well as with the observed particle-hole asymmetry in the DOS. Finally, we find that the phonon modes are softened over a broad range of wave vectors, and that including the presence of strong phonon fluctuations leads to a suppression of the transition temperature, implying a range of intermediate temperatures dominated by incoherent, fluctuating, charge order and a corresponding pseudogap. In light of these findings, and the generic nature of the electronic structure of $\mathrm{NbSe}_{2}$, we argue that the momentum and orbital dependence of the electron-phonon coupling may be expected to play a similarly central part in the description of other charge-ordered materials, especially in the presence of relatively strong coupling as in the case of the layered high- $T_{\mathrm{C}}$ superconductors.

\section{Results}

The extent of electronic nesting. $\mathrm{NbSe}_{2}$ is a layered material, in which hexagonal layers of niobium atoms are sandwiched between similar layers of selenium atoms, displaced so that they lie above and below half of the $\mathrm{Nb}$ interstitial locations, as shown in the inset of Fig. 1. Consecutive sandwich layers are displaced to have the complementary half of the interstices occupied, giving two formula units per unit cell. The Fermi surface consists of two concentric barrel-shaped pockets centred around both the $\Gamma$ and the $K$ points, as well as a very small pancake-shaped pocket surrounding $\Gamma$, which we ignore in the analysis below. The inner pockets at $K$ are seen in ARPES to develop the largest CDW gaps $^{4,10}$. The CDW itself is of the $3 Q$ type, with three equivalent superposed modulations at $120^{\circ}$ with respect to one another.

To describe the electronic states, we employ a Slater-Koster tight-binding fit to the band structure using a basis of Se- $4 p$ and $\mathrm{Nb}-4 d$ orbitals ${ }^{31,32}$. For the two main bands crossing $E_{\mathrm{F}}$ that give rise to the barrel-shaped pockets (which we will refer to as inner, developing the $\mathrm{CDW}$ instability, and outer), the fit is constrained by data from ARPES measurements ${ }^{10}$, while the remaining 20 bands are fit to recent LDA calculations ${ }^{5}$. In agreement with earlier reports ${ }^{32}$, we find that both the inner and outer bands at $E_{\mathrm{F}}$ are primarily composed of the $d_{3 z^{2}-r^{2}}$ orbitals centred on the two $\mathrm{Nb}$ atoms within a unit cell, in the form of bonding (inner) and antibonding (outer) combinations (as shown in Fig. 1). 
a

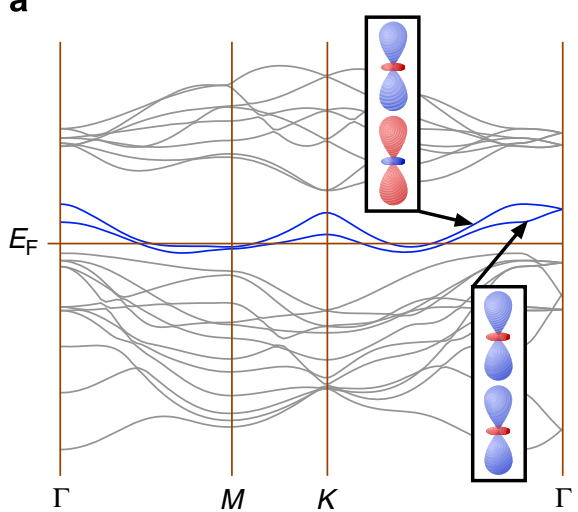

b

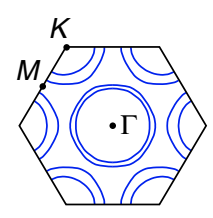

c

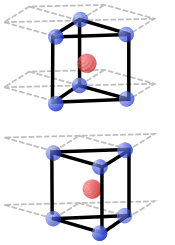

Figure 1 | Model band structure of $\mathbf{N b S e}_{2}$. (a) The band structure of $\mathrm{NbSe}_{2}$ modelled by a tight-binding fit to both ARPES data and firstprinciples band structure calculations. The two bands crossing $E_{\mathrm{F}}$ (thick lines) are the ones involved in the formation of charge order. They consist of bonding and antibonding combinations of the two niobium $d_{3 z^{2}-r^{2}}$ orbitals within a unit cell, as indicated (with red and blue signifying postive and negative parts of the orbital wave functions). (b) The Fermi surface resulting from the two bands crossing $E_{\mathrm{F} .}$ (c) The layered atomic structure of $\mathrm{NbSe}_{2}$, with $\mathrm{Nb}$ in red and $\mathrm{Se}$ in blue. The unit cell, containing two formula units across consecutive sandwich layers, is indicated.

Focussing on the two non-interacting bands at $E_{\mathrm{F}}$, we introduce the interaction between electrons $\psi$ and phonons $\varphi$ :

$$
\hat{H}_{\text {int }}=\sum_{m, n} \sum_{\mathbf{k}, \mathbf{q}} g_{\mathbf{k}, \mathbf{k}+\mathbf{q}}^{m, n} \hat{\varphi}_{\mathbf{q}} \hat{\psi}_{\mathbf{k}}^{\dagger m} \hat{\psi}_{\mathbf{k}+\mathbf{q}}^{n} .
$$

The bare phonon dispersion can be described by a Brillouin function tending to a maximum of $11.2 \mathrm{meV}$ at the zone boundary ${ }^{19}$. The electron-phonon coupling $g$ depends on the momenta, as well as the band indices $m$ and $n$, of the the ingoing and outgoing electron states. Following Varma et al. ${ }^{33}$, we model both of these dependencies using knowledge purely of the electronic structure, which has been shown to work well for a range of transition metals and their compounds. In terms of the matrices of tight-binding overlap integrals $S_{\mathbf{k}}$ and generalized eigenvectors $A_{\mathbf{k}}$, the electron-phonon coupling matrix elements are given by ${ }^{33}$ :

$$
\mathbf{g}_{\mathbf{k} \mathbf{k}^{\prime}}^{m n}=\mathbf{v}_{\mathbf{k}}^{m}\left[A_{\mathbf{k}}^{\dagger} S_{\mathbf{k}} A_{\mathbf{k}^{\prime}}\right]^{m n}-\left[A_{\mathbf{k}}^{\dagger} S_{\mathbf{k}^{\prime}} A_{\mathbf{k}^{\prime}}\right]^{m n} \mathbf{v}_{\mathbf{k}^{\prime}}^{n},
$$

where a constant prefactor has been omitted, and the band velocity $\mathbf{v}^{n}$ for electrons in band $n$ is defined in terms of the band energy $\xi_{\mathbf{k}}^{n}$ as $\mathbf{v}_{\mathbf{k}}^{n}=\partial \xi_{\mathbf{k}}^{n} / \partial \mathbf{k}$. In the interaction of equation (1) we restrict attention to the longitudinal component of $\mathbf{g}$, since only the longitudinal acoustic phonons are observed to soften in inelastic neutron and X-ray scattering experiments ${ }^{19}$.

Inserting the tight-binding results into equation (2), we find that the interband electron-phonon coupling is strictly zero, while the intraband coupling is about a factor of three stronger for the inner band than for the outer. This result immediately explains why the experimentally observed CDW gap is so much more pronounced in the inner band ${ }^{4,10}$, since the static electronic susceptibility (that is, the linear response to lattice distortions) scales with the square of the electron-phonon coupling:

$$
\chi_{0}(\mathbf{q}) \propto \sum_{\mathbf{k}}\left|g_{\mathbf{k}, \mathbf{k}+\mathbf{q}}^{m n}\right| \frac{{ }^{2} f\left(\xi_{\mathbf{k}}^{m}\right)-f\left(\xi_{\mathbf{k}+\mathbf{q}}^{n}\right)}{\xi_{\mathbf{k}+\mathbf{q}}^{n}-\xi_{\mathbf{k}}^{m}} .
$$

Here $f(\xi)$ is the Fermi-Dirac distribution function, and the static Lindhard function is given by $\chi_{0}$ when $g=1$. The absence of interband coupling causes the contributions of the two bands to

a

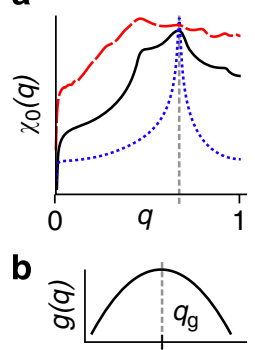

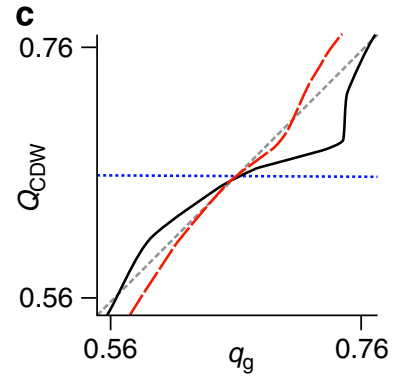

Figure 2 | Quantifying the extent of nesting in $\mathbf{N b S e}_{2}$. (a) Electronic susceptibility as a function of momentum transfer along $\Gamma M$. Here $q$ is defined through $\mathbf{q}=q \Gamma M$, with $q \in[0,1]$. Blue dotted line: in a perfectly nested 1D band structure, the susceptibility is sharply peaked at $\mathbf{q}=\mathbf{Q}_{\mathrm{CDW}}$. Red dashed curve: the result for the two bands in $\mathrm{NbSe}_{2}$ crossing $E_{\mathrm{F}}$ and an orbital-independent electron-phonon coupling. The susceptibility is nearly flat, without any peaks. Black solid curve: the susceptibility of just the inner band, in which a CDW gap is observed. Although a clear maximum can be seen at $\mathbf{Q}_{\mathrm{CDW}}$, it is not a sharp divergence indicative of perfect nesting. (b) The model electron-phonon coupling $g=-a\left|\mathbf{q}_{\mathrm{g}}-\mathbf{q}\right|^{2}+g_{\max }$ as $\mathbf{q}$ is varied along $\Gamma M$. (c) Evolution of the CDW vector $\mathbf{Q}_{\mathrm{CDW}}$ with varying peak position $\mathbf{q}_{\mathrm{g}}$ of the model electron-phonon coupling. Blue dotted line: in a perfectly nested 1D band structure, the electronic structure determines the CDW formation and $\mathbf{Q}_{\mathrm{CDW}}$ is independent of $\mathbf{q}_{\mathrm{g}}$. Red dashed curve: the result for an orbital-independent electron-phonon coupling and the two bands crossing $E_{\mathrm{F}}$, which is close to a straight line at $45^{\circ}$, indicative of a negligible role of the electronic structure. Black solid curve: the evolution relevant for $\mathrm{NbSe}_{2}$, given by just the inner band as imposed by the orbitaldependent electron-phonon coupling. This curve interpolates between the flat plateau of a perfectly nested structure, and the $45^{\circ}$ line dominated by just the structure of the electron-phonon coupling.

the susceptibility to be entirely independent. This means that the lower value of the intraband coupling in the outer band leads to an order of magnitude suppression of its contribution to $\chi_{0}$ and therefore to a correspondingly reduced gap in its Fermi surface pocket. It is thus the orbital character of the electronic bands, and consequently the orbital dependence of the electron-phonon coupling, that causes a relative size difference between the CDW gaps in the pockets around the $K$-point, even if they are equally well nested.

The expression for the susceptibility in equation (3) can further be used to quantify both the amount of nesting present in $\mathrm{NbSe}_{2}$ and to evaluate the relative contribution of the electron-phonon coupling matrix elements in selecting the observed value of the (incommensurate) ordering wave vector $\mathbf{Q}_{\mathrm{CDW}}=(1-\delta) \frac{2}{3} \Gamma M$, with $\delta \approx 0.02^{1}$. In the left panel of Fig. 2 , three susceptibilities are compared. The dotted blue line shows the susceptibility for a perfectly nested model with a single 1D band of cosine dispersion. The sharp peak characteristic of a nested Fermi surface is apparent. The dashed red line is the bare susceptibility in $\mathrm{NbSe}_{2}$, obtained by setting $g=1$ in equation (3). This does not include any of the momentum and orbital dependence of the electronphonon coupling and is the form that has been widely used in previous studies $s^{5,10,15}$. It is almost completely flat except for a very broad and low maximum far from $\mathbf{Q}_{\mathrm{CDW}}$, and can therefore not explain the observed ordering vector. The solid black line, on the other hand, displays the susceptibility of just the inner band. A broad but distinct maximum develops at the experimentally observed CDW vector. The selection of the ordering vector is thus shown to arise from the combined influence of both the electronic structure and the orbital and momentum-dependent electron-phonon coupling, with neither contribution being negligible ${ }^{4,10}$. 
Restricting attention to the inner band, we can quantify the extent to which nesting contributes to the selection of $\mathbf{Q}_{\mathrm{CDW}}$. For this purpose we temporarily switch to a simplified form of $g$, which is a function only of the momentum transfer: $g=-a\left(q_{\mathrm{g}}-q\right)^{2}+g_{\max }$. Even though in general the shape of the susceptibility is determined by both the incoming momentum and the momentum transfer, modelling the electron-phonon coupling in this simplified form allows us to directly quantify the effect of varying the position of its peak, and thus its influence on the position of the peak in $\chi_{0}$. The values of the parameters $a, q_{\mathrm{g}}$ and $g_{\max }$ can be estimated by fitting the experimentally observed phonon softening ${ }^{7}$ to the expression for the renormalized phonon energy in the random phase approximation (RPA), $\Omega_{\mathrm{RPA}}^{2}=\Omega_{0}\left(\Omega_{0}-\chi_{0}\right)$, where we take $\Omega_{0}$ to be the high-temperature (unrenormalized) dispersion. In the right panel of Fig. 2 we plot the variation of the maximum in the susceptibility (that is, the expected $\mathbf{Q}_{\mathrm{CDW}}$ ) as $\mathbf{q}_{\mathrm{g}}$ is varied along $\Gamma M$. Again, the blue dotted line represents the situation of a perfectly nested band structure, in which the electron-phonon coupling never overcomes the peak in the susceptibility, and thus has no effect on $\mathbf{Q}_{\mathrm{CDW}}$. The red dashed line has the same form of $g$ for all bands, and is close to a straight line at $45^{\circ}$ (grey dashed line). A true $45^{\circ}$ line would indicate that $\mathbf{Q}_{\mathrm{CDW}}=\mathbf{q}_{\mathrm{g}}$, implying that the CDW vector is completely determined by the electronphonon coupling, with the electronic structure having no influence at all. The solid black line, which shows the behaviour of just the inner band, interpolates between these two extremes. There is no plateau, but there is an indication of some flattening, suggesting that while it is mainly the momentum dependence of the electron-phonon coupling that determines the value of $\mathrm{CDW}$-ordering vector, the role of the electronic structure cannot be neglected entirely.

The CDW gap. Having identified the importance of both electron-phonon coupling and electronic structure, we now revert to using the full expression of equation (2). To compare the size and shape of the CDW gap resulting from the orbital- and momentum-dependent $g$ to the experimentally observed behaviour, the RPA gap equations shown diagrammatically in Fig. 3 are solved self-consistently. We simplify the calculation by using the $9 \times 9$ gap matrix which includes all higher harmonics appropriate for a commensurate $\mathrm{CDW}$ wave vector at $\mathbf{Q}=\frac{2}{3} \Gamma M$, as an approximation for the gap matrix of the incommensurate $\mathrm{CDW}$ in $\mathrm{NbSe}_{2}$ with $\mathbf{Q}_{\mathrm{CDW}} \simeq 0.98 \frac{2}{3} \Gamma M$. We further fix the values of the electronic self-energies to $7 \mathrm{meV}$, approximating the self-consistent result across $\mathbf{k}$ in the high-temperature phase, and constrain the momentum dependence of the gap function to a five parameter tight-binding fit consistent with the symmetries of the lattice. A self-consistent solution for the gap function to all orders in RPA can then be found by searching for a fixed point in the flow of consecutive iterations of the gap equation.
Figure 4 shows energy and momentum cuts through the electronic spectral functions resulting from the solution to the gap equations, as well as their corresponding DOS. A good match with the shape of the experimentally determined DOS in STS experiments can be achieved by introducing a $4 \mathrm{meV}$ shift in the chemical potential, which falls well within the range of experimental uncertainty $^{15}$. The overall strength of the electron-phonon coupling, the constant of proportionality omitted in equation (2) and the only free parameter in the theory, is set to give a maximum CDW gap magnitude of $\approx 12 \mathrm{meV}$. The Fermi surface on the right of Fig. 4 then shows a CDW gap opening on the inner band only, and Fermi arcs forming at the same locations as observed in ARPES measurements ${ }^{4,10,23}$. The extent of the gap on the Fermi surface, its size (apparent from the dispersion's back-folding in the centre of Fig. 4) and its restriction to one band are all in agreement with experimental findings ${ }^{4,10,23}$. The plot of the DOS on the left indicates that these results, which were previously interpreted to imply a gap size of $\sim 5 \mathrm{meV}^{4,10}$, are in fact consistent with the STS results indicating a particle-hole asymmetric gap of $\sim 12 \mathrm{meV}^{15,23}$. The asymmetry hides most of the gap structure above $E_{\mathrm{F}}$, where photoemission intensity is suppressed by the Fermi-Dirac distribution $^{23}$. The comparison between the experimental STS data and the calculated RPA results shows that the particle-hole asymmetry arises naturally from the interplay between the electronic structure and the momentum-dependent electronphonon coupling.

The pseudogap. The central role of the orbital- and momentumdependent electron-phonon coupling in determining both the CDW propagation vector and the structure of the gap suggests that the charge order in $\mathrm{NbSe}_{2}$ falls in the strong-coupling regime, in which the electronic structure alone is not sufficient to characterize the emergent charge order. In the presence of strong electron-phonon coupling, it is well known that the entropy associated with localized lattice vibrations plays an essential part in the system's thermal evolution ${ }^{20}$. To understand the properties of $\mathrm{NbSe}_{2}$ above its CDW transition temperature, it is therefore necessary to consider the influence of phonon fluctuations beyond RPA. The so-called mode-mode coupling approximation (MMA) includes the lowest order terms of this type and has been shown to successfully describe the influence of phonon fluctuations in some of the electronic properties of related dichalcogenide materials like $\mathrm{TaSe}_{2}$ and $\mathrm{TiSe}_{2}{ }^{21,22,34}$. The additional terms included in the MMA fall into two categories: a vertex correction, which we neglect by appeal to Migdal's theorem because of the large momentum transfer associated with $\mathbf{Q}_{\mathrm{CDW}}$, and self-energy corrections to the internal electron lines (indicated diagrammatically in Fig. 5), which we include. In agreement with recent $\mathrm{X}$-ray scattering observations on $\mathrm{NbSe}_{2}{ }_{7,19}$, the MMA-renormalized phonon spectrum shown in Fig. $5 \mathrm{~b}$ shows a broad range of phonon modes softening near the

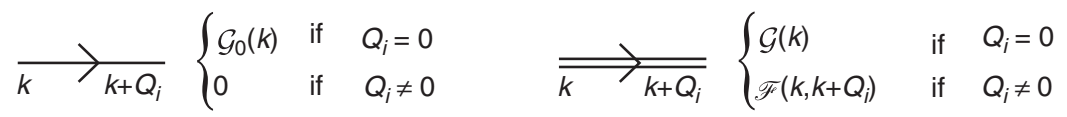

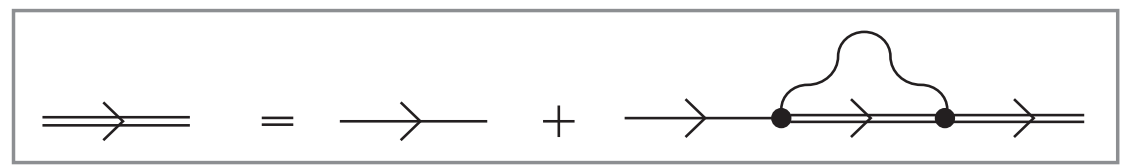

Figure 3 | The RPA gap equation. Diagrammatic form of the self-consistent RPA gap equations. As indicated in the legend, the single arrows indicate bare electronic Green's functions $\mathcal{G}_{0}$, and the double arrows the renormalized Green's functions $\mathcal{G}$ and $\mathscr{F}$. The anomalous functions $\mathscr{F}\left(k, k+Q_{i}\right)=\left\langle\hat{\psi}_{k} \hat{\psi}_{k}^{\dagger}+Q_{i}\right\rangle$ do not conserve crystal momentum, and correspond to the CDW order parameter. Notice that the momentum labels for the Green's functions include Matsubara frequencies, and the final diagram includes a summation over the anomalous momentum $Q_{i}$ of the internal electronic propagator. 
a

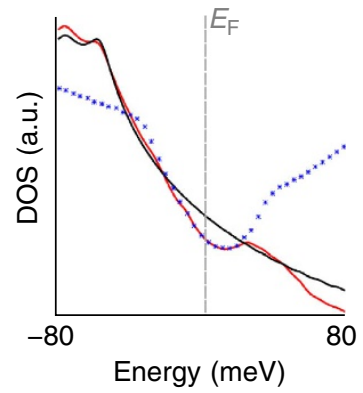

b

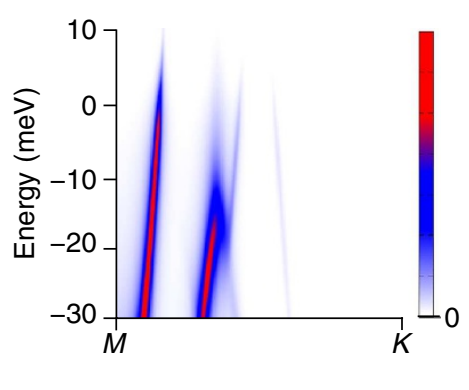

C

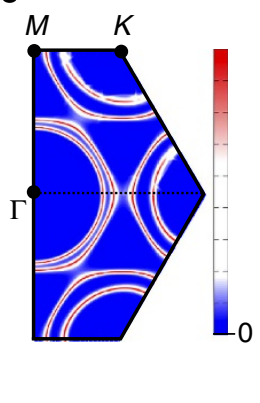

Figure 4 | Comparison to experimental results. (a) The calculated density of states for the bare dispersion (black line) and renormalized dispersion (red line) compared with experimental results from STS measurements (blue points) ${ }^{15}$. The opening of the CDW gap leads to an asymmetric suppression of the density of states, centred above $E_{\mathrm{F}}$ (b) The dispersion of the calculated spectral functions along $M K$, with the colour scale indicating intensity in arbitrary units. The back-bending induced by the gap at $E_{\mathrm{F}}$ is apparent in the band corresponding to the inner pocket about $K$. (c) Fermi energy cut of the spectral function resulting from the self-consistent gap equations, showing the formation of Fermi arcs as a result of gaps opening along MK. Again the colour scale indicates intensity in arbitrary units. Below the dashed line the bare bands are shown for comparison. Note that the outer band develops no discernible gap; this can be compared directly with the results of recent ARPES measurements ${ }^{4,10}$

a

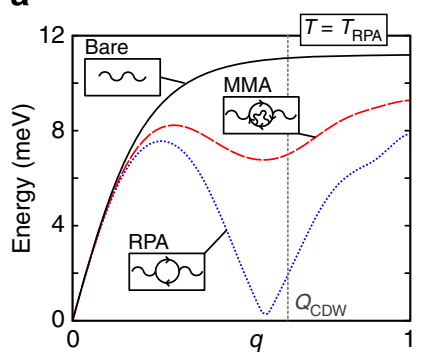

b

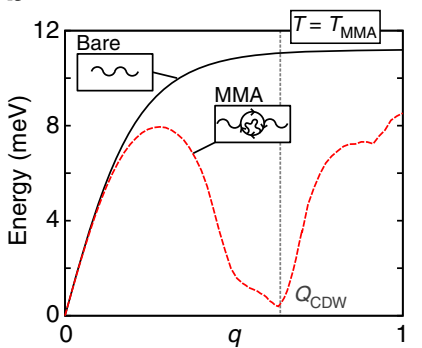

Figure 5 | Phonon dispersion in $\mathbf{N b S e}_{\mathbf{2}}$. (a) The solid black line indicates the bare phonon dispersion as a function of momentum along $\Gamma M$, based on high-temperature $\mathrm{X}$-ray scattering results ${ }^{19}$. Here $q$ is defined through $\mathbf{q}=q \Gamma M$, with $q \in[0,1]$. The blue dotted curve shows the dispersion resulting from the (mean field) RPA calculation at $T_{\text {RPA }}=303.5 \mathrm{~K}$, while the red dashed curve represents the MMA prediction at the same temperature (including the phonon fluctuations as shown in the diagrammatical representation of the renormalized phonon propagator). Both calculations are based on the same tight-binding band structure. The fluctuations of the phonon field can be seen to suppress the onset of charge order predicted by the RPA theory. (b) The MMA dispersion at $T_{M M A}=33.5 \mathrm{~K}$, which represents the true transition temperature. It can be seen that the momentum of the softest phonon mode corresponds to the experimentally observed CDW propagation vector.

transition temperature, rather than the sharp Kohn anomaly indicative of well-nested materials. The momentum of the softest phonon mode at the transition temperature coincides with the experimentally observed CDW propagation vector.

The softening of phonon modes, as the charge-ordered state is approached from high temperatures, is suppressed by the fluctuations beyond RPA. Since the temperature at which the first phonon mode reaches zero energy defines the transition temperature $T_{\mathrm{CDW}}$, this means that the charge-ordering transition temperature in MMA, $T_{\mathrm{MMA}}$, is suppressed with respect to the corresponding value in RPA, $T_{\mathrm{RPA}}$. Choosing the overall strength of the electron-phonon coupling such that $T_{\mathrm{MMA}}$ matches the experimentally observed value of $T_{\mathrm{CDW}}=33.5 \mathrm{~K}$, Fig. 5 shows that the corresponding RPA transition occurs already at $T_{\mathrm{RPA}} \approx 300 \mathrm{~K}$. Physically, this suppression of the transition temperature can be ascribed to the presence of fluctuations in the phonon field. In the temperature range $T_{\mathrm{MMA}}<T<T_{\mathrm{RPA}}$, the phonon fluctuations are strong enough to destroy the long-range order predicted by (mean field) RPA theory. In that regime, the amplitude of the static order parameter vanishes, $\left\langle\hat{\varphi}_{\mathbf{Q}}\right\rangle=0$, but dynamic fluctuations of the order parameter persist, $\left\langle\left|\hat{\varphi}_{\mathbf{Q}}\right|^{2}\right\rangle>0^{20-22}$. The result is a locally fluctuating short-range ordered state, characterized by the presence of a non-zero order parameter amplitude, without any long-range phase coherence ${ }^{23}$. Due to the non-zero amplitude, the gap in the electronic structure will survive even above the transition temperature $T_{\mathrm{CDW}}=T_{\mathrm{MMA}}$, in the form of a pseudogap phase ${ }^{4,23}$. The dynamical fluctuations in this regime may become locally pinned in the presence of defects, creating islands of order up to $T=T_{\mathrm{RPA}} \approx 300 \mathrm{~K}$, in agreement with recent STM and X-ray scattering experiments ${ }^{23,24}$. The properties of this regime are reminiscent of the features that characterize the pseudogap phase of cuprate high-temperature superconductors ${ }^{35}$, which have recently been shown typically to contain charge-ordered phases of their $\mathrm{own}^{28-30}$. Specific similarities are the presence of a pseudogap, Fermi surface arcs, dynamical fluctuations and a corresponding sensitivity to the timescales employed by experimental probes, as well as the existence of the crossover to the normal state at $T^{*}=T_{\mathrm{RPA}}$. Note that the existence of a crossover temperature $T^{*} \approx 300 \mathrm{~K}$, between the pseudogap and disordered regimes predicted here, falls outside of the temperature range that has been probed in $\mathrm{NbSe}_{2}$ to date ${ }^{4,24}$.

\section{Discussion}

Although various theories for the formation of charge order in $\mathrm{NbSe}_{2}$ have been able to explain many parts of its diverse experimental signatures, the material has so far evaded the simultaneous description of all of its properties by a single consistent theoretical account. The main features, which remain difficult to reconcile with the weak-coupling theories considered so far, include the emergence of CDW order in spite of a lack of nesting in the electronic structure, and an anomalously flat electronic susceptibility, the softening of a broad range of phonon modes rather than a sharp Kohn anomaly, a discrepancy in the sizes of the CDW gap observed in different electron pockets in spite of them being similarly nested, disagreement about the existence, size and particle-hole symmetry of the CDW gap as seen by different experimental probes and the presence of a pseudogap phase characterized by locally fluctuating charge order above the CDW transition temperature. We have shown here that all of these observations can be consistently understood, within a single theory, to result from the simultaneous influence of the presence of a strong electron-phonon coupling, its orbital dependence and its dependence on both incoming and outgoing 
electron momentum. We have shown that none of these ingredients can be neglected in the full description of $\mathrm{NbSe}_{2}{ }_{5,21,22,34}$, suggesting their possible importance in other dichalcogenide materials as well.

The combination of both the momentum-dependent coupling and the particular electronic structure of $\mathrm{NbSe}_{2}$ is found to be crucial in the selection of the charge-ordering vector, while the orbital dependence naturally explains the different behaviours of different Fermi surface pockets. The same dependencies on momentum and orbital character are also reflected in the emergence of a particle-hole asymmetric gap, which has a size of about $12 \mathrm{meV}$, centred above $E_{\mathrm{F}}$ where it is largely inaccessible to observation by ARPES. These observations put $\mathrm{NbSe}_{2}$ in the regime of strongly coupled charge-ordered materials, in the sense that the specifics of the electron-phonon coupling qualitatively affect the properties of the ordered state.

The pseudogap phase above $T_{\mathrm{CDW}}$ may arise in such a scenario from local fluctuations of the lattice, which destroy the long-range phase coherence of the CDW order parameter but not its amplitude. Taking into account higher-order phonon fluctuations, we find that indeed the mean field transition temperature is suppressed, leading to a regime above $T_{\mathrm{CDW}}$ characterized by the absence of long-range charge order and the presence of phonon fluctuations. This agrees with the experimental observation of Fermi arcs and a pseudogap above the transition temperature, as well as that of pinned charge order at high temperatures around local defects. All of these signatures of the pseudogap phase are reminiscent of similar features seen in the pseudogap phase of cuprate high-temperature superconductors.

In conclusion, while the Peierls mechanism can explain charge ordering in 1D systems entirely in terms of the electronic structure, this is not the natural starting point for higherdimensional materials. Instead, it is necessary to include full information about the dependence of the electron-phonon coupling on the momenta and orbital characters of the electronic states involved in the CDW formation. This is evidenced by the present case of $\mathrm{NbSe}_{2}$, a typical quasi-two-dimensional material without a strongly nested Fermi surface, in which knowledge about all aspects of the electron-phonon coupling proves to be essential in explaining the full range of experimental observations. We suggest this consideration to be generically applicable across charge-ordered quasi-two-dimensional and quasi-three-dimensional materials lacking strong nesting features. In particular, the full momentum and orbital dependence of the electron-phonon coupling should be taken into account throughout the class of transition metal dichalcogenides and related material classes, including high-temperature superconductors.

\section{References}

1. Wilson, J. A., Di Salvo, F. J. \& Mahajan, S. Charge-density waves in metallic, layered, transition-metal dichalcogenides. Phys. Rev. Lett. 32, 882-885 (1974).

2. Peierls, R. More Surprises in Theoretical Physics (Princeton University Press, 1991).

3. Doran, N. J. A calculation of the electronic response function in $2 \mathrm{H}-\mathrm{NbSe} 2$ including electron-phonon matrix element effects. J. Phys. C 11, L959-L962 (1978).

4. Borisenko, S. V. et al. Two energy gaps and Fermi-surface "arcs" in NbSe2. Phys. Rev. Lett. 102, 166402 (2009).

5. Johannes, M. D., Mazin, I. I. \& Howells, C. A. Fermi-surface nesting and the origin of the charge-density wave in $\mathrm{NbSe}_{2}$. Phys. Rev. B73, 205102 (2006).

6. Rice, T. M. \& Scott, G. K. New mechanism for a charge-density-wave instability. Phys. Rev. Lett. 35, 120-123 (1975).

7. Weber, F. et al. Extended phonon collapse and the origin of the charge-density wave in 2H-NbSe2. Phys. Rev. Lett. 107, 107403 (2011).

8. Hess, H., Robinson, R. \& Waszczak, J. STM spectroscopy of vortex cores and the flux lattice. Physica B 169, 422-431 (1991).

9. Wang, C., Giambattista, B., Slough, C. G., Coleman, R. V. \& Subramanian, M. A. Energy gaps measured by scanning tunneling microscopy. Phys. Rev. B 42, 8890-8906 (1990)
10. Rahn, D. J. et al. Gaps and kinks in the electronic structure of the superconductor $2 \mathrm{H}-\mathrm{NbSe} 2$ from angle-resolved photoemission at $1 \mathrm{~K}$. Phys. Rev. B 85, 224532 (2012)

11. Straub, T. et al. Charge-density-wave mechanism in $2 \mathrm{H}-\mathrm{NbSe}_{2}$ : photoemission results. Phys. Rev. Lett. 82, 4504-4507 (1999).

12. Harper, J., Geballe, T. \& Salvo, F. D. Heat capacity of $2 \mathrm{H}-\mathrm{NbSe} 2$ at the charge density wave transition. Phys. Lett. A 54, 27-28 (1975).

13. Grüner, G. The dynamics of charge-density waves. Rev. Mod. Phys. 60, 1129-1181 (1988).

14. Moncton, D. E., Axe, J. D. \& DiSalvo, F. J. Study of superlattice formation in $2 \mathrm{H}-\mathrm{NbSe}_{2}$ and $2 \mathrm{H}-\mathrm{TaSe}_{2}$ by neutron scattering. Phys. Rev. Lett. 34, 734-737 (1975).

15. Soumyanarayanan, A. et al. Quantum phase transition from triangular to stripe charge order in NbSe2. Proc. Natl Acad. Sci. USA 110, 1623-1627 (2013).

16. Rainer, D. \& Sauls, J. A. inSuperconductivity: From Basic Physics to the Latest Developments. (eds Butcher, P. N. \& Lu, Y.) Ch. 2 45-78 (World Scientific, 1995).

17. Wang, Y. et al. Weak-coupling $d$-wave BCS superconductivity and unpaired electrons in overdoped $\mathrm{La}_{2}{ }_{-} \mathrm{Sr}_{\mathrm{x}} \mathrm{CuO}_{4}$ single crystals. Phys. Rev. B 76, 064512 (2007).

18. Hashimoto, M., Vishik, I. M., He, R.-H., Devereaux, T. P. \& Shen, Z.-X. Energy gaps in high-transition-temperature cuprate superconductors. Nat. Phys. 10, 483-495 (2014).

19. Weber, F. et al. Optical phonons and the soft mode in $2 \mathrm{H}-\mathrm{NbSe}_{2}$. Phys. Rev. B 87, 245111 (2013).

20. McMillan, W. L. Landau theory of charge-density waves in transition-metal dichalcogenides. Phys. Rev. B 12, 1187-1196 (1975).

21. Varma, C. M. \& Simons, A. L. Strong-coupling theory of charge-density-wave transitions. Phys. Rev. Lett. 51, 138-141 (1983).

22. Yoshiyama, H., Takaoka, Y., Suzuki, N. \& Motizuki, K. Effects on lattice fluctuations on the charge-density-wave transition in transition-metal dichalcogenides. J. Phys. C 19, 5591-5606 (1986).

23. Chatterjee, U. et al. Emergence of coherence in the charge-density wave state of 2H-NbSe2. Nat. Commun. 6, 6313 (2015).

24. Arguello, C. J. et al. Visualizing the charge density wave transition in $2 \mathrm{H}-\mathrm{NbSe} 2$ in real space. Phys. Rev. B 89, 235115 (2014).

25. Emery, V. J., Kivelson, S. A. \& Tranquada, J. M. Stripe phases in hightemperature superconductors. Proc. Natl Acad. Sci. USA 96, 8814-8817 (1999).

26. Wu, T. et al. Magnetic-field-induced charge-stripe order in the hightemperature superconductor YBa2Cu3Oy. Nature 477, 191-194 (2011).

27. Walstedt, R. E., Mason, T. E., Aeppli, G., Hayden, S. M. \& Mook, H. A. Pseudogap term in the magnetic response of cuprate superconductors. Phys. Rev. B 84, 024530 (2011).

28. Chang, J. et al. Direct observation of competition between superconductivity and charge density wave order in $\mathrm{YBa}_{2} \mathrm{Cu}_{3} \mathrm{O}_{6.67}$. Nat. Phys. 8, 871-876 (2012).

29. Ghiringhelli, G. et al. Long-range incommensurate charge fluctuations in $(\mathrm{Y}, \mathrm{Nd}) \mathrm{Ba} 2 \mathrm{Cu} 3 \mathrm{O}(6+\mathrm{x})$. Science 337, 821-825 (2012).

30. $\mathrm{Wu}, \mathrm{T}$. et al. Incipient charge order observed by NMR in the normal state of YBa2Cu3Oy. preprint at http://arxiv.org/abs/1404.1617 (2014).

31. Slater, J. C. \& Koster, G. F. Simplified LCAO method for the periodic potential problem. Phys. Rev. 94, 1498-1524 (1954).

32. Doran, N. J., Titterington, D. J., Ricco, B. \& Wexler, G. A tight binding fit to the bandstructure of $2 \mathrm{H}-\mathrm{NbSe}_{2}$ and $\mathrm{NbS}_{2}$. J. Phys. C 11, 685-698 (1978).

33. Varma, C. M., Blount, E. I., Vashishta, P. \& Weber, W. Electron-phonon interactions in transition metals. Phys. Rev. B 19, 6130-6141 (1979).

34. Inglesfield, J. E. Bonding and phase transitions in transition metal dichalcogenide layer compounds. J. Phys. C 13, 17-36 (1980).

35. Timusk, T. \& Statt, B. The pseudogap in high-temperature superconductors: an experimental survey. Rep. Prog. Phys. 62, 61-122 (1999).

\section{Acknowledgements}

We thank M. R. Norman for suggesting the approach discussed here and for numerous insightful discussions. J.v.W. acknowledges support from a VIDI grant financed by the Netherlands Organisation for Scientific Research (NWO).

\section{Author contributions}

Both authors contributed equally to all aspects of the work.

\section{Additional information}

Competing financial interests: The authors declare no competing financial interests.

Reprints and permission information is available at http://npg.nature.com/ reprintsandpermissions/

How to cite this article: Flicker F. et al. Charge order from orbital-dependent coupling evidenced by $\mathrm{NbSe}_{2}$. Nat. Commun. 6:7034 doi: 10.1038/ncomms8034 (2015). 